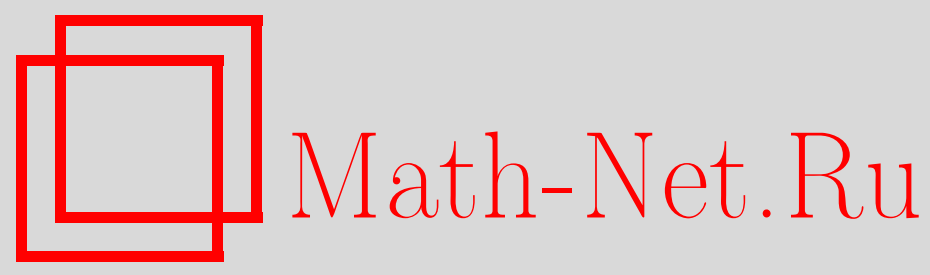

Ю. С. Семенов, О графах и кольцах Ли, Матем. заметки, 2005, том 77, выпуск 3, 449-459

DOI: https://doi.org/10.4213/mzm2505

Использование Общероссийского математического портала Math-Net.Ru подразумевает, что вы прочитали и согласны с пользовательским соглашением http://www .mathnet.ru/rus/agreement

Параметры загрузки:

IP : 52.87 .193 .239

26 апреля 2023 г., 17:32:10

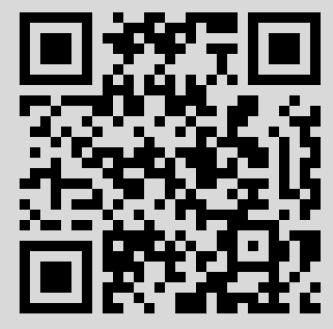




\title{
О ГРАФАХ И КОЛЬЦАХ ЛИ
}

\author{
Ю. С. Семёнов
}

По конечному ориентированному графуу Г естественным образом строятся конечномерные градуированные нильпотентные кольца Ли $\mathfrak{l}(\Gamma)$ и $\mathfrak{g}(\Gamma)$, связанные соответственно с поддеревьями и связными подграфами в $Г$. Предложены также различные варианты этих конструкций. Кроме того, указано вложение колец Ли вида $\mathfrak{l}(\Gamma)$ в присоединенные кольца Ли конечномерных ассоциативных колец, также определяемых графом $\Gamma$.

Библиографиял: 4 названия.

\section{Введение}

По-видимому, все нильпотентные конечномерные алгебры Ли невозможно классифицировать в полном объеме. В настоящей работе предлагается несколько достаточно естественных конструкций, позволяющих связать с конечным ориентированным графом нильпотентное градуированное кольцо (алгебру) Ли. В действительности эти кольца с точностью до изоморфизма не зависят от ориентации графа.

Идея построения таких колец Ли возникла при работе над доказательством изоморфизма целочисленных гомологий свободной нильпотентной групшы класса 2 c $r$ свободными порождающими и свободного нильпотентного кольца Ли класса 2 с тем же числом свободных порождающих (см. [1]).

Изложим вкратце основные полученные результаты. Пусть $Г$ - конечньй ориентированньй граф, под которым подразумевается симплициальный 1-комплекс, т.е. кратные ребра и петли не допускаются. Множества $\Gamma^{0}$ и $\Gamma^{1}-$ это множества вершин и ребер графа Г соответственно.

Обозначим через $\mathscr{T}(\Gamma)$ множество всех поддеревьев в Г (связных подграфов без циклов). В частности, в $\mathscr{T}(\Gamma)$ есть деревья, которые являются вершинами или ребрами, соединяющими две вершины. Отметим, что если Г распадается в объединение своих связных компонент $\Gamma_{1} \sqcup \cdots \sqcup \Gamma_{m}$, то

$$
\mathscr{T}(\Gamma)=\mathscr{T}\left(\Gamma_{1}\right) \sqcup \cdots \sqcup \mathscr{T}\left(\Gamma_{m}\right) .
$$

Обозначим через $\mathfrak{l}(\Gamma)$ свободньй $\mathbb{Z}$-модуль с базисом $\mathscr{T}(\Gamma)$, т.е. элементами $\mathfrak{l}(\Gamma)$ являются целочисленные линейные комбинации деревьев из $\mathscr{T}(\Gamma)$. Пусть $T_{1}, T_{2} \in \mathscr{T}(\Gamma)$ и $T_{1} \cap T_{2}=\varnothing$. В этом случае $E\left(T_{1}, T_{2}\right)$ обозначает множество ориентированных ребер, идущих из $T_{1}$ в $T_{2}$ (при этом $\left.E\left(T_{1}, T_{2}\right) \cap E\left(T_{2}, T_{1}\right)=\varnothing\right)$. Если $e \in E\left(T_{1}, T_{2}\right) \sqcup E\left(T_{2}, T_{1}\right)$, 
то под $T_{1} \# T_{2} T_{2}$ подразумевается связная сумма деревьев $T_{1}, T_{2}$ над $e$, т.е. дерево, получаемое присоединением к $T_{1}$ и $T_{2}$ ребра $e$. Обратим внимание, что $T_{1} \#{ }_{e} T_{2}=T_{2} \#{ }_{e} T_{1}$, а число вершин в $T_{1} \# e_{2} T_{2}$ равно сумме числа вершин в $T_{1}$ и $T_{2}$.

Определим операцию умножения $\left[T_{1}, T_{2}\right]$ на деревьях $T_{1}, T_{2} \in \mathfrak{l}(\Gamma)$ следующим обра3ом:

$$
\left[T_{1}, T_{2}\right]= \begin{cases}\sum_{e \in E\left(T_{1}, T_{2}\right)} T_{1} \# T_{2}-\sum_{e \in E\left(T_{2}, T_{1}\right)} T_{1} \#{ }_{e} T_{2}, & \text { если } T_{1} \cap T_{2}=\varnothing \\ 0, & \text { если } T_{1} \cap T_{2} \neq \varnothing\end{cases}
$$

и распространим эту операцию по линейности на $\mathfrak{l}(\Gamma)$.

ПРЕДЛОЖЕНИЕ 1. Введенное умножение превращает $\mathfrak{l}(\Gamma)$ в кольцо Ли.

Помимо доказательства этого предложения в первом разделе мы отмечаем такие свойства кольца $\mathfrak{l}(\Gamma)$, как нильпотентность и градуированность, рассматриваем некоторые примеры, показываем функториальность этой конструкции (предложение 2). Если граф Г связен и имеет $n$ вершин, то ступень нильпотентности $\mathfrak{l}(\Gamma)$ в точности равна $n$ (предложение 3 ). Кроме того, предлагается ряд обобщений кольца $\mathfrak{l}(\Gamma)$. В частности, при построении можно брать не деревья, а связные подграфы в $Г$, что приводит к более общему кольцу $\mathfrak{g}(\Gamma)$, содержащему $\mathfrak{l}(\Gamma)$ в качестве подкольца (см. п. 1.6.1).

Однако центральную роль для нас играет кольцо $\mathfrak{l}(\Gamma)$, поскольку во втором разделе мы связьваем с графом $Г$ определенное конечномерное ассоциативное кольцо $\mathscr{A}(\Gamma)$ и строим, следуя идеям [1], вложение именно кольца $\mathfrak{l}(\Gamma)$ в соответствующее $\mathscr{A}(\Gamma)$ присоединенное кольцо Ли (предложение 4).

Автор выражает признательность профессору Ю. А. Бахтурину за полезные замечания.

\section{1. Кольца Ли, ассоциированные с графами}

1.1. Доказательство предложения 1. Доказательство совсем элементарно и сводится к рутинной проверке тождества Якоби. Равенство $\left[T_{1}, T_{2}\right]=-\left[T_{2}, T_{1}\right]$ очевидно следует из определения умножения, поскольку при перестановке $T_{1}$ и $T_{2}$ множества ребер $E\left(T_{1}, T_{2}\right)$ и $E\left(T_{2}, T_{1}\right)$ переходят друг в друга.

Тождество Якоби достаточно проверить для трех деревьев $T_{1}, T_{2}$ и $T_{3}$ таких, что

$$
T_{1} \cap T_{2}=T_{2} \cap T_{3}=T_{3} \cap T_{1}=\varnothing
$$

Действительно, если, например, $T_{3} \cap T_{1} \neq \varnothing$, то любое дерево вида $T_{1} \# T_{2}$ (или $\left.T_{3} \#{ }_{e} T_{2}\right)$ нетривиально пересекается с $T_{3}\left(\right.$ с $\left.T_{1}\right)$, и тождество Якоби выполнено тривиальньгм образом.

Рассмотрим

$$
\left[\left[T_{1}, T_{2}\right], T_{3}\right]+\left[\left[T_{2}, T_{3}\right], T_{1}\right]+\left[\left[T_{3}, T_{1}\right], T_{2}\right]
$$

и, в частности, первое слагаемое этой суммы. Тогда

$$
\left[\left[T_{1}, T_{2}\right], T_{3}\right]=\sum_{e \in E\left(T_{1}, T_{2}\right)}\left[T_{1} \# e_{e} T_{2}, T_{3}\right]-\sum_{e \in E\left(T_{2}, T_{1}\right)}\left[T_{1} \# e_{e} T_{2}, T_{3}\right]
$$




$$
\begin{aligned}
& =\sum_{\substack{e \in E\left(T_{1}, T_{2}\right) \\
f \in E\left(T_{1}, T_{3}\right)}}\left(T_{1} \#{ }_{e} T_{2}\right) \#{ }_{f} T_{3}+\sum_{\substack{e \in E\left(T_{1}, T_{2}\right) \\
f \in E\left(T_{2}, T_{3}\right)}}\left(T_{1} \# e_{e} T_{2}\right) \#_{f} T_{3} \\
& -\sum_{\substack{e \in E\left(T_{1}, T_{2}\right) \\
f \in E\left(T_{3}, T_{1}\right)}}\left(T_{1} \# e_{e} T_{2}\right) \#_{f} T_{3}-\sum_{\substack{e \in E\left(T_{1}, T_{2}\right) \\
f \in E\left(T_{3}, T_{2}\right)}}\left(T_{1} \#{ }_{e} T_{2}\right) \#_{f} T_{3} \\
& -\sum_{\substack{e \in E\left(T_{2}, T_{1}\right) \\
f \in E\left(T_{1}, T_{3}\right)}}\left(T_{1} \# e_{e} T_{2}\right) \#_{f} T_{3}-\sum_{\substack{e \in E\left(T_{2}, T_{1}\right) \\
f \in E\left(T_{2}, T_{3}\right)}}\left(T_{1} \#{ }_{e} T_{2}\right) \#_{f} T_{3} \\
& +\sum_{\substack{e \in E\left(T_{2}, T_{1}\right) \\
f \in E\left(T_{3}, T_{1}\right)}}\left(T_{1} \# e_{e} T_{2}\right) \#_{f} T_{3}+\sum_{\substack{e \in E\left(T_{2}, T_{1}\right) \\
f \in E\left(T_{3}, T_{2}\right)}}\left(T_{1} \#{ }_{e} T_{2}\right) \#_{f} T_{3} .
\end{aligned}
$$

Остальные два слагаемых в (1) записьваются аналогичньп образом. В результате получится 24 групшы слагаемых, которые, как нетрудно убедиться, попарно сократятся. Например, в $\left[\left[T_{3}, T_{1}\right], T_{2}\right]$ есть группа слагаемых

$$
-\sum_{\substack{f \in E\left(T_{1}, T_{3}\right) \\ e \in E\left(T_{1}, T_{2}\right)}}\left(T_{1} \#_{f} T_{3}\right) \#_{e} T_{2}=-\sum_{\substack{e \in E\left(T_{1}, T_{2}\right) \\ f \in E\left(T_{1}, T_{3}\right)}}\left(T_{1} \#_{e} T_{2}\right) \#_{f} T_{3},
$$

сокращающаяся с первой группой слагаемых в правой части (2).

1.2. Простейшие свойства кольца $\mathfrak{l}(\Gamma)$. Пусть $\Gamma=\Gamma_{1} \sqcup \cdots \sqcup \Gamma_{m}$, где $\Gamma_{j}-$ связная компонента графа $Г$. Поскольку меж ду различными связными компонентами нет ни одного ребра, имеет место разложение $\mathfrak{l}(\Gamma)$ в прямую сумму колец Ли:

$$
\mathfrak{l}(\Gamma)=\bigoplus_{j=1}^{m} \mathfrak{l}\left(\Gamma_{j}\right)
$$

С другой стороны,

$$
\mathfrak{l}(\Gamma)=\bigoplus_{k=1}^{n} \mathfrak{l}_{k}(\Gamma)
$$

где $\mathbb{Z}$-подмодуль $\mathfrak{l}_{k}(\Gamma)$ порождается всеми деревьями, у которых ровно $k$ вершин, $n-$ число вершин в графе $Г$. Из определения умножения в $\mathfrak{l}_{k}(\Gamma)$ непосредственно вытекает, что

$$
\left[\mathfrak{l}_{i}(\Gamma), \mathfrak{l}_{j}(\Gamma)\right] \subseteq \mathfrak{l}_{i+j}(\Gamma)
$$

т.е., во-первых, кольцо Ли $\mathfrak{l}(\Gamma)$ градуировано, а, во-вторых, оно нильпотентно ступени $\leqslant n=\left|\Gamma^{0}\right|$ (в предложении 3 будет доказано, что для связного графа $Г$ ступень нильпотентности кольца Ли $\mathfrak{l}(\Gamma)$ в точности равна $n$ - числу вершин $\Gamma)$. В частности, $\mathbb{Z}$-подмодули

$$
\mathfrak{j}_{p}(\Gamma)=\bigoplus_{k=p}^{n} \mathfrak{l}_{k}(\Gamma), \quad p=2, \ldots, n,
$$

- это идеалы кольца $\mathfrak{l}(\Gamma)$. 


\section{3. Примеры.}

ПРИмеР 1. Пусть граф̆ Г имеет $n$ вершин и ни одного ребра. Тогда $\mathfrak{l}(\Gamma) \cong \mathbb{Z}^{n}-$ абелево кольцо Ли.

ПРИмеР 2. Рассмотрим более содержательньй пример. Пусть $\Gamma=\Gamma(n)$ - полньй ориентированньй граф с $n$ вершинами и $\left(\begin{array}{c}n \\ 2\end{array}\right)$ ребрами. Для определенности будем считать, что вершины занумерованы натуральными числами от 1 до $n$, а началом ориентированного ребра, которое соединяет $i$-ю и $j$-ю вершины, служит вершина с меньшим номером. Подсчитаем $\mathbb{Z}$-ранг $\mathfrak{l}(\Gamma)$. Любое дерево с $k$ вершинами однозначно определяет некоторое подмножество мошности $k$ в множестве вершин графа $Г$. Число деревьев в $Г$ с фиксированньми $k$ вершинами находится по известной формуле Кэли (см. [2], а также, например, [3], [4]) и равно $k^{k-2}$. Отсюда

$$
\operatorname{rk}_{\mathbb{Z}} \mathfrak{l}(\Gamma)=\sum_{k=1}^{n}\left(\begin{array}{l}
n \\
k
\end{array}\right) k^{k-2}
$$

ПРИмеР 3. Пусть $\mathfrak{u t}(n+1, \mathbb{Z})$ - кольцо Ли верхних строго треугольных матриц порядка $n+1$ над $\mathbb{Z}$ со стандартным базисом $E_{i j}, 1 \leqslant i<j \leqslant n+1$. Рассмотрим граф $\Lambda(n)$ следующего вида:

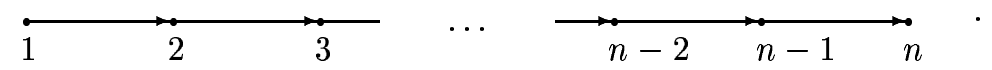

Изоморфизм между кольцами $\mathfrak{l}(\Lambda(n))$ и $\mathfrak{u t}(n+1, \mathbb{Z})$ строится следующим образом. Пусть $T_{i j}, 1 \leqslant i \leqslant j \leqslant n,-$ поддерево в $\Lambda(n)$ с вершинами в точках $i, j$. Поставим ему в соответствие матрицу $E_{i, j+1}$ и продолжим это отображение по $\mathbb{Z}$-линейности на $\mathfrak{l}(\Lambda(n))$. Нетрудно убедиться, что это и будет нужный изоморфизм.

1.4. Функториальность. Рассмотрим категорию конечных ориентированных графов $\mathscr{F} \mathscr{O} \mathscr{G}$, морфизмами в которой являются вложения графов (сохраняющие отношение инцидентности и ориентацию). Пусть $\Gamma, \Phi-$ два конечных ориентированных графа и $\iota: \Gamma \rightarrow \Phi$ - вложение. Мы будем отождествлять $\Gamma$ с его образом при вложении $\iota$, т.е. записывать $\Gamma \subseteq \Phi$. Легко видеть, что если $\Gamma \subseteq \Phi$, то множество деревьев $\mathscr{T}(\Gamma)$ является подмножеством в $\mathscr{T}(\Phi)$. Обозначим $\mathfrak{j}(\Gamma, \Phi) \mathbb{Z}$-подмодуль в $\mathfrak{l}(\Phi)$, порожденный всеми деревьями из дополнения $\mathscr{T}(\Phi) \backslash \mathscr{T}(\Gamma)$. Он является идеалом в $\mathfrak{l}(\Phi)$, что непосредственно вытекает из определения умножения деревьев.

Более того, точна следующая последовательность:

$$
0 \longrightarrow \mathfrak{j}(\Gamma, \Phi) \longrightarrow \mathfrak{l}(\Phi) \stackrel{\pi}{\longrightarrow} \mathfrak{l}(\Gamma) \longrightarrow 0
$$

где $\pi=\pi_{\Gamma}^{\Phi}-$ это эпиморфизм, для которого

$$
\pi(T)=\left\{\begin{array}{l}
0, \quad \text { если } T \in \mathscr{T}(\Phi) \backslash \mathscr{T}(\Gamma), \\
T, \quad \text { если } T \in \mathscr{T}(\Gamma) .
\end{array}\right.
$$

Укажем достаточно простое условие расщепимости последовательности (3). Пусть из того, что две произвольные вершины $v_{1}, v_{2} \in \Gamma \subseteq \Phi$ соединены ребром в графе $\Phi$, 
вытекает, что это ребро принадлежит подграфу Г (соответствующее вложение графов назовем полным $)$. Тогда $\mathbb{Z}$-линейное отображение $s: \mathfrak{l}(\Gamma) \rightarrow \mathfrak{l}(\Phi)$ такое, что $s(T)=T$ $\forall T \in \mathscr{T}(\Gamma)$, является вложением колец Ли, расщепляющим $(3)$, т.е. $\pi s=1_{\mathfrak{l}(\Gamma)}$.

Пусть $\Gamma \subseteq \Phi \subseteq \Delta$. Очевидно, что композиция морфизмов $\pi_{\Gamma}^{\Phi} \pi_{\Phi}^{\Delta}$ равна $\pi_{\Gamma}^{\Delta}$. Если $\mathrm{id}: \Gamma \rightarrow \Gamma$ - тождественное вложение, то соответствующий гомоморфизм колец Ли $\pi$ является тождественным. Это означает, что доказано следующее предложение.

ПРЕДЛОЖЕНИЕ 2. Соответствие Г $\longrightarrow \mathfrak{l}(\Gamma)$ является контравариантным фун-

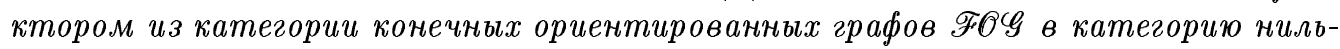
потентных колеи, Ли.

В качестве следствия докажем следующее

ПРЕДЛОЖЕНИЕ 3. Пусть Г - связный граф с $n$ вершинами. Тогда ступень нильпотентности кольца $\mathfrak{l}(\Gamma)$ в точности равна $n$.

ДокАЗАТЕЛьСтво. Выберем в Г максимальное дерево $M$. В виду эпиморфизма $\mathfrak{l}(\Gamma) \rightarrow \mathfrak{l}(M)$ достаточно доказать утверждение для кольца $\mathfrak{l}(M)$. Пусть $v_{1}, \ldots, v_{n}-$ все вершины $M$, выписанные в таком порядке, что для всех $k=1, \ldots, n-1$ вершина $v_{k+1}$ соединена ребром с поддеревом, порожденным в $M$ вершинами $v_{1}, \ldots, v_{k}$ (это всегда можно сделать в силу связности $M)$. Индукцией по $k$ легко получить, что в кольце $\mathfrak{l}(M)$ левонормированный коммутатор $\left[\left[\left[v_{1}, v_{2}\right], \ldots\right], v_{k}\right]$ равен с точностью до знака \pm 1 дереву, порожденному в $M$ вершинами $v_{1}, \ldots, v_{k}$. В частности, при $k=n$ получим $\left[\left[\left[v_{1}, v_{2}\right], \ldots\right], v_{n}\right]= \pm M \neq 0$, что и требовалось доказать.

1.5. Изменение ориентации ребер. Покажем, что на самом деле кольцо $\mathfrak{l}(\Gamma) \mathrm{c}$ точностью до изоморфизма не зависит от выбора ориентации графа Г. Достаточно показать, что при замене ориентации одного ребра $f$ на противоположную соответствующие кольца изоморфны. Пусть $\Gamma$ - исходньй граф, а $\Gamma^{*}-$ граф, в котором ребро $f$ ориентировано в противоположную сторону. Между множествами деревьев $\mathscr{T}(\Gamma)$ и $\mathscr{T}\left(\Gamma^{*}\right)$ имеется естественньй изоморфизм; дерево $T$, рассматриваемое как дерево в $\Gamma^{*}$, обозначим $T^{*}$. Пусть $\mathbb{Z}$-линейное отображение $\varphi: \mathfrak{l}(\Gamma) \rightarrow \mathfrak{l}\left(\Gamma^{*}\right)$ действует на элементах базиса по следуюшему правилу:

$$
\varphi(T)=\left\{\begin{aligned}
-T^{*}, & \text { если } f \in T, \\
T^{*}, & \text { если } f \notin T .
\end{aligned}\right.
$$

Равенство $\left[\varphi\left(T_{1}\right), \varphi\left(T_{2}\right)\right]=\varphi\left(\left[T_{1}, T_{2}\right]\right)$ очевидно, если $T_{1} \cap T_{2} \neq \varnothing$ или ребро $f$ не принадлежит ни $T_{1}$, ни $T_{2}$, ни множеству ребер $E\left(T_{1}, T_{2}\right) \sqcup E\left(T_{2}, T_{1}\right)$. Поэтому нужно разобрать лишг два случая. Если $f$ принадлежит, скажем, дереву $T_{1}$, то $\left[\varphi\left(T_{1}\right), \varphi\left(T_{2}\right)\right]=$ $\left[-T_{1}^{*}, T_{2}^{*}\right]$. С другой стороны, $f$ будет принадлежать любому дереву вида $T_{1} \#{ }_{e} T_{2}$, и значит, $\varphi\left(T_{1} \# e_{2} T_{2}\right)=-T_{1}^{*} \# e_{2}^{*}$, т.е. $\left[\varphi\left(T_{1}\right), \varphi\left(T_{2}\right)\right]=\varphi\left(\left[T_{1}, T_{2}\right]\right)$ и в этом случае.

Пусть теперь $f$ принадлежит, например, $E\left(T_{1}, T_{2}\right)$. Тогда противоположно ориентированное ребро $f^{*}$ будет принадлежать $E\left(T_{2}^{*}, T_{1}^{*}\right)$. При этом

$$
\begin{aligned}
\varphi\left(\left[T_{1}, T_{2}\right]\right) & =\sum_{e \in E\left(T_{1}, T_{2}\right)} \varphi\left(T_{1} \# e_{e} T_{2}\right)-\sum_{e \in E\left(T_{2}, T_{1}\right)} \varphi\left(T_{1} \# e_{e} T_{2}\right) \\
& =\varphi\left(T_{1} \# f_{f} T_{2}\right)+\sum_{\substack{e \in E\left(T_{1}, T_{2}\right) \\
e \neq f}} \varphi\left(T_{1} \#{ }_{e} T_{2}\right)-\sum_{e \in E\left(T_{2}, T_{1}\right)} \varphi\left(T_{1} \#{ }_{e} T_{2}\right)
\end{aligned}
$$




$$
\begin{aligned}
& =-T_{1}^{*} \# f^{*} T_{2}^{*}+\sum_{e \in E\left(T_{1}^{*}, T_{2}^{*}\right)} T_{1}^{*} \# e_{e} T_{2}^{*}-\sum_{\substack{e \in E\left(T_{2}^{*}, T_{1}^{*}\right) \\
e \neq f^{*}}} T_{1}^{*} \# e_{2}^{*} \\
& =\left[T_{1}^{*}, T_{2}^{*}\right]=\left[\varphi\left(T_{1}\right), \varphi\left(T_{2}\right)\right] .
\end{aligned}
$$

\section{6. Модификации конструкции.}

1.6.1. Кольцо $\mathfrak{g}(\Gamma)$. В предложенной конструкции можно везде заменить деревья (т.е. связные подграфы без ииклов) на связные подграфы. При этом все рассуждения проходят точно так же, как и в случае деревьев. Будем обозначать множество связных подграфов в $Г$ через $\mathscr{G}(\Gamma)$, а соответствующее кольцо Ли через $\mathfrak{g}(\Gamma)$.

Пусть $\Phi \in \mathscr{G}(\Gamma)$ и пусть $b_{1}(\Phi)=\left|\Phi^{1}\right|-\left|\Phi^{0}\right|+1-$ первое число Бетти графа $\Phi$. Если $\Phi_{1} \cap \Phi_{2}=\varnothing$, то $b_{1}\left(\Phi_{1} \#{ }_{e} \Phi_{2}\right)=b_{1}\left(\Phi_{1}\right)+b_{1}\left(\Phi_{2}\right)$, что позволяет ввести еще одну градуировку на кольце $\mathfrak{g}(\Gamma)$ помимо градуировки по числу вершин:

$$
\mathfrak{g}(\Gamma)=\bigoplus_{q \geqslant 0} \mathfrak{g}_{q}(\Gamma)
$$

где $\mathbb{Z}$-подмодуль $\mathfrak{g}_{q}(\Gamma)$ порожден всеми $\Phi \in \mathscr{G}(\Gamma)$ с условием $b_{1}(\Phi)=q$. В частности, подкольцо $\mathfrak{g}_{0}(\Gamma)$ - это в точности кольцо $\mathfrak{l}(\Gamma)$.

1.6.2. Случай бесконечного графа Г. Можно не ограничиваться конечньпи графами $\Gamma$ (однако по-прежнему требуется, чтобы деревья $T \in \mathscr{T}(\Gamma)$ или подграфы $\Phi \in \mathscr{G}(\Gamma)$ были конечньми). В этом случае кольца $\mathfrak{g}(\Gamma)$ и $\mathfrak{l}(\Gamma)$ будут, по крайней мере, локально нильпотентньми.

1.6.3. Кольца Ли и размеченные графы. Пусть $R$ - коммутативное ассоциативное кольцо, $\Gamma$ - ориентированный графф и $\lambda: \Gamma^{1} \rightarrow R$ - функция меток ребер. Используя ранее введенные обозначения, определим $\mathfrak{g}(\Gamma, \lambda, R)$ как свободньй $R$-модуль с базисом $\mathscr{G}(\Gamma)$ и умножением, задаваемьм на элементах базиса по правилу

$$
\left[\Phi_{1}, \Phi_{2}\right]= \begin{cases}\sum_{e \in E\left(\Phi_{1}, \Phi_{2}\right)} \lambda(e) \Phi_{1} \# \Phi_{2}-\sum_{e \in E\left(\Phi_{2}, \Phi_{1}\right)} \lambda(e) \Phi_{1} \# \Phi_{2}, & \text { если } \Phi_{1} \cap \Phi_{2}=\varnothing, \\ 0, & \text { если } \Phi_{1} \cap \Phi_{2} \neq \varnothing .\end{cases}
$$

Это умножение превращает $\mathfrak{g}(\Gamma, \lambda, R)$ в кольцо Ли над $R$, что доказьвается совершенно аналогично предложению 1 . Функториальность $\mathfrak{g}(\Gamma, \lambda, R)$ по $Г$ имеет место при условии, что при вложении ориентированных графов сохраняются метки ребер. Кольцо $\mathfrak{g}(\Gamma)$ - это не что иное, как $\mathfrak{g}\left(\Gamma, \lambda_{1}, \mathbb{Z}\right)$, где $\lambda_{1} \equiv 1$. Если имеется вложение колец $R_{1} \hookrightarrow R_{2}$, то естественно рассматривать $\mathfrak{g}\left(\Gamma, \lambda, R_{1}\right)$ как подкольцо Ли в $\mathfrak{g}\left(\Gamma, \lambda, R_{2}\right)$ (расширение кольца скаляров).

Однако отметим, что если $\Gamma$ - связньй граф с $n$ вершинами, то, вообше говоря, ступень нильпотентности кольца $\mathfrak{g}(\Gamma, \lambda, R)$ может быть и строго меньше $n$, например, в случае, когда все метки ребер нулевые.

\section{2. Об одном вложении кольца $\mathfrak{l}(\Gamma)$}

Цель данного раздела - построить вложение кольца Ли $\mathfrak{l}(\Gamma)$ в присоединенное кольцо Ли некоторого конечномерного ассоциативного кольца. 
2.1. Кольцо $\mathscr{A}(\Gamma)$. Пусть $\Gamma$ - конечный ориентированный граф. Рассмотрим следующее ассоциативное кольцо с единищей $\mathscr{A}(\Gamma)$, связанное с графом $\Gamma$. Как $\mathbb{Z}$-модуль $\mathscr{A}(\Gamma)$ свободно порождается всеми элементами вида $a(E, V)$, где $E, V$ - некоторые подмножества множеств ориентированных ребер $\Gamma^{1}$ и вершин $\Gamma^{0}$ соответственно. Умножение в $\mathscr{A}(\Gamma)$ задается на базисных элементах по формуле (где $E\left(V_{1}, V_{2}\right)$ обозначает множество всех ориентированных ребер с началом в $V_{1}$ и концом в $\left.V_{2}\right)$ :

$$
a\left(E_{1}, V_{1}\right) a\left(E_{2}, V_{2}\right)=a\left(E_{1} \cup E_{2} \cup E\left(V_{1}, V_{2}\right), V_{1} \sqcup V_{2}\right),
$$

если $V_{1} \cap V_{2}=\varnothing$, иначе произведение полагается равньм 0. Несложно проверяется ассоциативность умножения:

$$
\begin{aligned}
& \left(a\left(E_{1}, V_{1}\right) a\left(E_{2}, V_{2}\right)\right) a\left(E_{3}, V_{3}\right) \\
& \quad=a\left(E_{1}, V_{1}\right)\left(a\left(E_{2}, V_{2}\right) a\left(E_{3}, V_{3}\right)\right) \\
& \quad=a\left(E_{1} \cup E_{2} \cup E_{3} \cup E\left(V_{1}, V_{2}\right) \cup E\left(V_{1}, V_{3}\right) \cup E\left(V_{2}, V_{3}\right), V_{1} \sqcup V_{2} \sqcup V_{3}\right)
\end{aligned}
$$

в том случае, когда $V_{1} \cap V_{2}=V_{1} \cap V_{3}=V_{2} \cap V_{3}=\varnothing$ (если это не так, то получается 0$)$. Единицей кольца $\mathscr{A}(\Gamma)$ является элемент $a(\varnothing, \varnothing)=1$. Заметим, что

$$
\operatorname{rk}_{\mathbb{Z}} \mathscr{A}(\Gamma)=2^{\left|\Gamma^{0}\right|+\left|\Gamma^{1}\right|} .
$$

Из определения следует, что $a(E, V)=a(E, \varnothing) a(\varnothing, V)$, и элементы $a(E, \varnothing)$ всегда лежат в центре кольца $\mathscr{A}(\Gamma)$. В дальнейшем элементы вида $a(E, \varnothing)$ будем обозначать просто через $a(E)$ (в частности, $a(\{e\}, \varnothing)=a(e)$ для любого ребра $e$ ), а элементы вида $a(\varnothing, V)$ через $a(V)$.

Пусть граф Г обладает дополнительньм свойством: вершинам можно приписать метки $1,2, \ldots, n=\left|\Gamma^{0}\right|$ так, что началом любого ориентированного ребра является вершина с меньшей меткой. Другими словами, $\Gamma$ является подграфом полного графа $\Gamma(n)$, который рассматривался нами в примере 2. Это ограничение совершенно не существенно, так как этого всегда можно добиться путем изменения ориентации некоторых ребер, что приводит к изоморфному кольцу Ли $l(\Gamma)$ (см. п. 1.5).

Покажем, что имеется вложение

$$
\alpha: \mathfrak{l}(\Gamma) \longrightarrow[\mathscr{A}(\Gamma)]
$$

$([\mathscr{A}(\Gamma)]$ - присоединенное кольцо Ли). Для определения $\alpha$ нам понадобятся элементы $\mu(T)$ (называемые метками деревьев) для каждого дерева $T \in \mathscr{T}(\Gamma)$, которые в общем виде представляются в виде произведения $a\left(e_{1}\right) \cdots a\left(e_{p}\right)\left(e_{1}, \ldots, e_{p}\right.$ - некоторые ребра, зависящие от $T)$. K описанию этих элементов мы и приступаем.

2.2. Метки деревьев. Будем считать, что вершины $v_{1}, \ldots, v_{n}$ графа $\Gamma$ упорядочены в соответствии с естественным порядком индексов, а ориентированные ребра $e=e_{i j}$, которые соединяют вершины $v_{i}$ и $v_{j}$, - лексикографически, т.е. $e_{i j}<e_{k l}$, если либо $j<l$, либо $j=l$, но при этом $i<k$. Пусть, как и раньше, $T$ - некоторое дерево в $\mathscr{T}(\Gamma)$. Добавление к $T$ еще одного ребра определяет в Г некоторый цикл (простую петлю) $Z$. Фиксируем на нем ориентацию в соответствии с направлением, которое указывает старшее ребро цикла. Если $f$ - произвольное ребро цикла $Z$, то оно также определяет на $Z$ 
некоторую ориентацию. Назовем ребро $f$ положительным относительно $Z$, если эта ориентация совпадает с той, которую мы выбрали.

Определим теперь метку $\mu(T)$ дерева $T$. Обозначим через $[T]$ подграфф в $\Gamma$, порожденный всеми вершинами дерева $T$. Положим $\mu(T)=\prod_{e} a(e)$, где произведение берется по всем ребрам $e \in[T] \backslash T$, которые положительны относительно возникающих при их добавлении циклов.

ПримеРЫ. Пусть $\Gamma=\Gamma(n)$ - полньй граф и $T$ - звездное дерево с ребрами $e_{1 i}$, $i=2, \ldots, n$. Тогда все дополнительные ребра положительны относительно соответствуюших циклов, поэтому

$$
\mu(T)=\prod_{1<i<j \leqslant n} a\left(e_{i j}\right)
$$

- произведение, взятое по всем дополнительным ребрам. Напротив, если $T=\Lambda(n)-$ простая цепь с ребрами $e_{i, i+1}, i=1, \ldots, n-1$, из примера 3 , то ни одно дополнительное ребро не является положительным и $\mu(T)=1$.

2.3. Построение вложения. Положим

$$
\alpha(T)=a\left(T^{0}\right) \mu(T) \prod_{e \in T}(a(e)-1)
$$

и продолжим это отображение по линейности на $\mathfrak{l}(\Gamma)$.

ПРЕДЛОЖЕНИЕ 4. Отображение $\alpha$ задает вложение кольиа Ли $\mathfrak{l}(\Gamma)$ в присоединенное ковио Ли $[\mathscr{A}(\Gamma)]$.

ДокАЗАТЕЛьСТво. Проверим, что вьполняется равенство

$$
\alpha\left(\left[T_{1}, T_{2}\right]\right)=\alpha\left(T_{1}\right) \alpha\left(T_{2}\right)-\alpha\left(T_{2}\right) \alpha\left(T_{1}\right)
$$

Если $T_{1} \cap T_{2} \neq \varnothing$, то $T_{1}$ и $T_{2}$ имеют общую вершину, и в силу равенства (5) обе части (6) равны 0 . Пусть $T_{1} \cap T_{2}=\varnothing$. Нам нужно показать, что

$$
\sum_{e \in E\left(T_{1}, T_{2}\right)} \alpha\left(T_{1} \# e T_{2}\right)-\sum_{e \in E\left(T_{2}, T_{1}\right)} \alpha\left(T_{1} \#{ }_{e} T_{2}\right)=\alpha\left(T_{1}\right) \alpha\left(T_{2}\right)-\alpha\left(T_{2}\right) \alpha\left(T_{1}\right) .
$$

Поскольку в $\mathscr{A}(\Gamma)$ вьполнены следуюшие соотношения:

$$
\begin{aligned}
& a\left(T_{1}^{0}\right) a\left(T_{2}^{0}\right)=a\left(T_{1}^{0} \sqcup T_{2}^{0}\right) \prod_{e \in E\left(T_{1}, T_{2}\right)} a(e), \\
& a\left(T_{2}^{0}\right) a\left(T_{1}^{0}\right)=a\left(T_{1}^{0} \sqcup T_{2}^{0}\right) \prod_{e \in E\left(T_{2}, T_{1}\right)} a(e),
\end{aligned}
$$

то (7) вытекает из равенства

$$
\begin{aligned}
& \sum_{e \in E\left(T_{1}, T_{2}\right)}(a(e)-1) \mu\left(T_{1} \# T T_{2}\right)-\sum_{e \in E\left(T_{2}, T_{1}\right)}(a(e)-1) \mu\left(T_{1} \#{ }_{e} T_{2}\right) \\
& =\mu\left(T_{1}\right) \mu\left(T_{2}\right) \prod_{e \in E\left(T_{1}, T_{2}\right)} a(e)-\mu\left(T_{2}\right) \mu\left(T_{1}\right) \prod_{e \in E\left(T_{2}, T_{1}\right)} a(e)
\end{aligned}
$$


Отметим, что в силу определения $\mu(T)$ любую метку $\mu\left(T_{1} \# e_{2} T_{2}\right)$ можно представить как произведение $\mu\left(T_{1}\right) \mu\left(T_{2}\right) \rho(e)$, где $\rho(e)$ состоит из произведения элементов вида $a(f)$, соответствующих некоторьм ребрам $f$ из $E\left(T_{1}, T_{2}\right) \sqcup E\left(T_{2}, T_{1}\right)$ (конкретньй вид $\rho(e)$ определен ниже, в лемме 1$)$, т.е. $\mu\left(T_{1} \# e_{2} T_{2}\right)$ всегда “делится" на $\mu\left(T_{1}\right) \mu\left(T_{2}\right)$.

Таким образом, (8) следует из равенства

$$
\sum_{e \in E\left(T_{1}, T_{2}\right)}(a(e)-1) \rho(e)-\sum_{e \in E\left(T_{2}, T_{1}\right)}(a(e)-1) \rho(e)=\prod_{e \in E\left(T_{1}, T_{2}\right)} a(e)-\prod_{e \in E\left(T_{2}, T_{1}\right)} a(e)
$$

которое мы и рассмотрим, но сначала сделаем некоторое отступление от доказательства предложения.

2.4. Упорядочение множества $E\left(T_{1}, T_{2}\right) \sqcup E\left(T_{2}, T_{1}\right)$. Пусть $e, f \in E\left(T_{1}, T_{2}\right) \sqcup$ $E\left(T_{2}, T_{1}\right)$ и $Z=Z(e, f)$ - цикл, которьй получается присоединением к $T_{1}, T_{2}$ ребер $e, f$. Скажем, что $e \prec f$, если при положительном обходе $Z$ (т.е. в направлении старшего ребра этого цикла) после прохождения ребра $e$ мы оказываемся в дереве $T_{2}$. Если это не так, то будем говорить, что $e \succ f$.

Покажем, что введенное отношение действительно является отношением порядка на множестве $E\left(T_{1}, T_{2}\right) \sqcup E\left(T_{2}, T_{1}\right)$, т.е. если $e \prec f, f \prec h$, то и $e \prec h$. Предположим, что $h \prec e$. Рассмотрим объединение всех ребер из $Z(e, f), Z(f, h), Z(h, e)$. Это некоторый графф $\Phi=\Phi(e, f, h) \subseteq \Gamma$, все вершины которого, кроме двух вершин степени 3 , имеют степень 2 , причем граф $\Phi$ остается связным при удалении произвольного ребра. Любое ребро $b$ граба $\Phi$ входит ровно в два из указанных выше трех циклов. Если $e \prec f, f \prec h$, $h \prec e$, то при последовательном положительном обходе всех трех циклов ребро $b$ будет проходиться ровно два раза: один раз в соответствии с ориентацией ребра $b$, другой раз - в противоположном направлении. Пусть $b$ - старшее ребро в графе $\Phi$ (относительно порядка всех ребер графа $\Gamma$ ). Очевидно, что $b$ - старшее ребро в обоих циклах, которым оно принадлежит. Поскольку положительный обход цикла всегда происходит в направлении старшего ребра цикла, мы получаем противоречие со сделанным предположением, т.е. $e \prec h$.

Таким образом, можно упорядочить все ребра из $E\left(T_{1}, T_{2}\right) \sqcup E\left(T_{2}, T_{1}\right)$. Вьпишем их в порядке возрастания: $e_{1} \prec \cdots \prec e_{t}$. Обозначим

$$
\rho_{21}(e)=\prod_{\substack{f \succ e \\ f \in E\left(T_{2}, T_{1}\right)}} a(f), \quad \rho_{12}(e)=\prod_{\substack{f \prec e \\ f \in E\left(T_{1}, T_{2}\right)}} a(f)
$$

ЛЕмма 1. В ранее введенных обозначениях $\rho(e)=\rho_{21}(e) \rho_{12}(e)$.

ДоказАтЕльство. Действительно, если ребро $е$ фиксировано (скажем, $e \in E\left(T_{1}\right.$, $\left.T_{2}\right)$ ), то $a(f)$ входит в метку $\rho(e)$ в двух случаях, а именно, если $f \succ e, f \in E\left(T_{2}, T_{1}\right)$ или $f \prec e, f \in E\left(T_{1}, T_{2}\right)$ (в этих случаях ребро $f$ положительно ориентировано относительно возникающего цикла $Z=Z(e, f))$.

Разложение $\rho(e)=\rho_{21}(e) \rho_{12}(e)$ будем называть полярным.

2.5. Продолжение доказательства предложения 4. То, что элементы $\rho(e)$ удовлетворяют (9), вытекает из следующей леммы. 
Лемма 2. Пусть $e_{1} \prec \cdots \prec e_{t}-$ все ребра из $E\left(T_{1}, T_{2}\right) \sqcup E\left(T_{2}, T_{1}\right) u$

$$
S(p)=\sum_{i=1}^{p} \operatorname{sgn} e_{i}\left(\rho\left(e_{i}\right)-\rho\left(e_{i}\right) a\left(e_{i}\right)\right), \quad p=1, \ldots, t
$$

әде $\operatorname{sgn} e_{i}=-1$, если $e_{i} \in E\left(T_{1}, T_{2}\right) ;$ иначе $\operatorname{sgn} e_{i}=1$. Тогда, если $e_{p} \in E\left(T_{1}, T_{2}\right)$, то

$$
S(p)=-\prod_{e \in E\left(T_{2}, T_{1}\right)} a(e)+\rho\left(e_{p}\right) a\left(e_{p}\right)
$$

а если $e_{p} \in E\left(T_{2}, T_{1}\right)$, mo

$$
S(p)=-\prod_{e \in E\left(T_{2}, T_{1}\right)} a(e)+\rho\left(e_{p}\right)
$$

ДокАЗАТЕЛЬСТво проведем индукцией по $p$. При $p=1$ утверждение непосредственно следует из равенства $\rho\left(e_{1}\right)=\rho_{21}\left(e_{1}\right)$. Пусть уже доказано, что

$$
S(p)=-\prod_{e \in E\left(T_{2}, T_{1}\right)} a(e)+\rho\left(e_{p}\right) a\left(e_{p}\right)
$$

(для определенности рассмотрим случай $e_{p} \in E\left(T_{1}, T_{2}\right)$; оставшийся случай рассматривается аналогично). Если $e_{p+1} \in E\left(T_{1}, T_{2}\right)$, то

$$
S(p+1)=-\prod_{e \in E\left(T_{2}, T_{1}\right)} a(e)+\rho\left(e_{p}\right) a\left(e_{p}\right)-\rho\left(e_{p+1}\right)+\rho\left(e_{p+1}\right) a\left(e_{p+1}\right) .
$$

Из полярного разложения $\rho(e)$ вытекает, что $\rho\left(e_{p}\right) a\left(e_{p}\right)=\rho\left(e_{p+1}\right)$, т.е. шаг индукции сделан. Аналогично исследуется случай, когда $e_{p+1} \in E\left(T_{2}, T_{1}\right)$. Лемма 2 доказана.

При $p=t$ в любом случае получается

$$
S(t)=\prod_{e \in E\left(T_{1}, T_{2}\right)} a(e)-\prod_{e \in E\left(T_{2}, T_{1}\right)} a(e) .
$$

Действительно, если $e_{t} \in E\left(T_{1}, T_{2}\right)$, то

$$
\rho\left(e_{t}\right) a\left(e_{t}\right)=\prod_{e \in E\left(T_{1}, T_{2}\right)} a(e),
$$

а иначе

$$
\rho\left(e_{t}\right)=\prod_{e \in E\left(T_{1}, T_{2}\right)} a(e) .
$$

Таким образом, равенство (9), а вместе с ним и гомоморфность отображения $\alpha$, доказаны. 
Убедимся в том, что гомоморфизм $\alpha$ является вложением. Пусть

$$
\sum_{T} n_{T} \alpha(T)=0, \quad n_{T} \in \mathbb{Z}
$$

где сумма берется по всем деревьям $T$ в $\Gamma$ с фиксированным множеством вершин $T^{0}=V$ (легко видеть, что общий случай сводится к этому). Из (11) и определения $\alpha$ следует, что в $\mathscr{A}(\Gamma)$ должно вьполняться равенство

$$
a(V) \sum_{T} n_{T} \mu(T) \prod_{e \in T}(a(e)-1)=0 .
$$

Поскольку

$$
\sum_{T} n_{T} \mu(T) \prod_{e \in T}(a(e)-1)=\sum k_{i} a\left(E_{i}\right),
$$

где $E_{i}$ - это подмножества множества ребер графа $\Gamma$, то

$$
a(V) \sum k_{i} a\left(E_{i}\right)=\sum k_{i} a\left(V, E_{i}\right)=0
$$

тогда и только тогда, когда $\sum k_{i} a\left(E_{i}\right)=0$.

Покажем, что в (12) все $n_{T}$ равны 0. Сократив на $a(V)$, получим

$$
\sum_{T} n_{T} \mu(T) \prod_{e \in T}(a(e)-1)=0,
$$

где правая часть рассматривается как элемент коммутативного подкольца $\mathscr{A}^{1}(\Gamma) \subset$ $\mathscr{A}(\Gamma)$, порожденного всеми $a(e)$. Обратим внимание, что для любого ребра $e$ выполняется $a(e)^{2}=a(e)$, и, как легко видеть, это и есть все определяющие соотношения кольца $\mathscr{A}^{1}(\Gamma)$. В частности, для любого дерева $T \in \mathscr{T}(\Gamma)$ можно определить гомоморфизм колец $\varphi_{T}: A^{1}(\Gamma) \rightarrow \mathbb{Z}$ такой, что

$$
\varphi_{T}(a(e))= \begin{cases}0, & \text { если } e \in T, \\ 1, & \text { если } e \notin T .\end{cases}
$$

Пусть $T$ - фиксированное дерево, учитываемое в сумме (13). Применим к равенству (13) гомоморфизм $\varphi_{T}$. В любом дереве $D$, отличном от $T$, есть ребро $f$, не входящее в $T$, и для которого $\varphi_{T}(a(f)-1)=0$. Поскольку $\varphi_{T}(\mu(T))=1$, из равенства $(13)$ вытекает, что $\pm n_{T}=0$. Таким образом, $\alpha$ действительно является вложением, и предложение 4 доказано.

\section{СПИСОК ЦИТИРОВАННОЙ ЛИТЕРАТУРЫ}

[1] Кузьмин Ю. В., Семёнов Ю. С. О гомологиях свободной нильпотентной группы класса 2 // Матем. сб. 1998. Т. 189. № 4. С. 49-82.

[2] Cayley A. Collected Mathematical Papers. Cambridge: Cambridge Univ. Press, 1889-1897.

[3] Clarke L. E. On Cayley's formula for counting trees // J. London Math. Soc. 1958. V. 33. P. 471-474.

[4] Moon J. W. Another proof of Cayley's formula for counting trees // Amer. Math. Monthly. 1963. V. 70. P. 846-847. 